\title{
Avulsion Fracture in Distal Phalanx of Left Great Toe: A Rare Unnoticed Karate Sports Injury
}

\author{
Md. Sumon Rahman a, d, Zakir Hossain ${ }^{\mathrm{b}}$, Hasan U1 Banna ${ }^{\mathrm{a}}$, \\ Mozammel Haque Milon ${ }^{\mathrm{c}}$
}

\begin{abstract}
Great toe injury is common in athletes. Besides violence, the sports injuries to the great toe vary according to the sharing force or impact. However, some great toe injuries can seriously affect the daily activities and some could also remain unnoticed. This case report documents an athlete with an unnoticed avulsion fracture of distal phalanx of the left great toe during karate practice on regular mat.
\end{abstract}

Keywords: Avulsion fracture; Sports injury; Hallux fracture

\section{Introduction}

Avulsion fracture occurs by the forceful pulling of a tendon or ligament with a piece of bone from its attachment. Avulsion fracture can happen anywhere in the body, but common sites include ankle, hip, clavicle, hand and foot irrespective of children or adults who involve in sports [1]. While fracture occurs with violent force or vigorous contraction of muscle, tendon or ligament that attaches to that part pulls that fragment away. Certain predisposing factors include [1]: 1) Children, for whom growth spurts could be a risk factor for avulsion fracture, and the children who used to play sports are at a greatest risk of developing such fracture; 2) Athletes, because certain sports involve high impact, ligament or muscle twisting and repeated changes in direction, the athlete (e.g. karate, mixed martial arts, gymnastics, high jump, heavy weight lifting, etc.) who experiences such impact or injury might have avulsion fracture; and 3) Dancers, who tend to put a lot of pressure on

Manuscript submitted December 13, 2018, accepted January 4, 2019

aDepartment of Surgery, Jahurul Islam Medical College \& Hospital, Bajitpur, Kishoregonj, Bangladesh

${ }^{b}$ Department of Orthopedics, Jahurul Islam Medical College \& Hospital, Bajitpur, Kishoregonj, Bangladesh

'Sensei, Shitokai Karate, Bangladesh Karate Federation (BKF), Dhaka, Bangladesh

${ }^{\mathrm{d} C o r r e s p o n d i n g ~ A u t h o r: ~ M d . ~ S u m o n ~ R a h m a n, ~ D e p a r t m e n t ~ o f ~ S u r g e r y, ~ J a h u r u l ~}$ Islam Medical College \& Hospital, Bajitpur 2336, Kishoregonj, Bangladesh. Email: drsumon@live.com

doi: https://doi.org/10.14740/jcs372 the feet and make frequent sudden movement, which cause avulsion fracture in feet.

The presentation of an avulsion fracture may vary but usually includes a history of a recent incident with sudden intense pain in and around the site of trauma. Affected part also develops swelling, bruising and trouble moving the bone or joint. It can be diagnosed by X-ray and sometimes it could also be misdiagnosed as muscle sprain and offered wrong treatment.

If treated properly, most of these injuries heal without any complication and surgical intervention is rarely necessary. But error in diagnosis and management can cause further displacement and nonunion. Improper management also leads to nerve irritation, chronic pain and gait dysfunction depending on the avulsion fracture site [2].

\section{Case Report}

A 35-year-old man, who is a specialized general surgeon used to practice karate as a regular workout, felt that his left great toe was stuck with the griping mat and developed sudden severe pain in his left great toe with difficulty to move interphalangeal (IP) joint while practicing frequent and repeated karate front kick (mae giri). After taking a rest the intensity of pain was reduced and he continued his practice and went home. The next day with nonsteroidal anti-inflammatory drug (NSAID) and cold compression he continued medical job, but

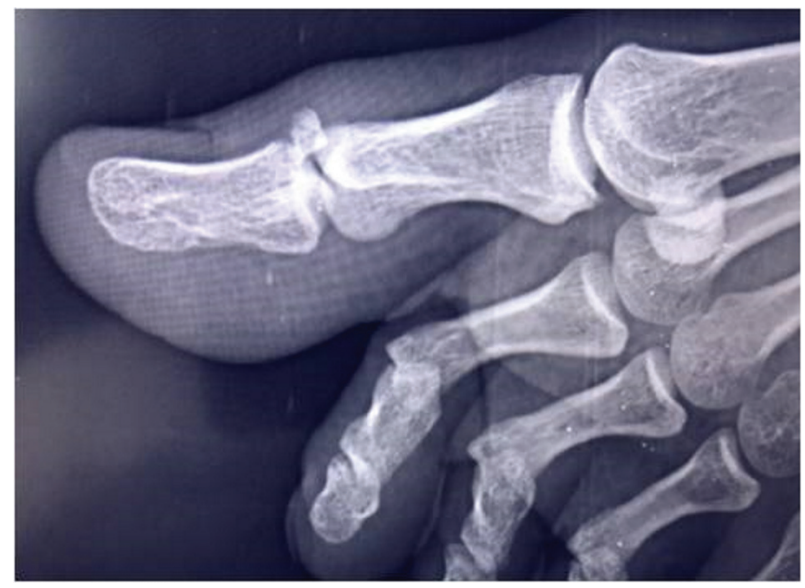

Figure 1. X-ray, lateral view of left hallux showing avulsion fracture of distal phalanx. 


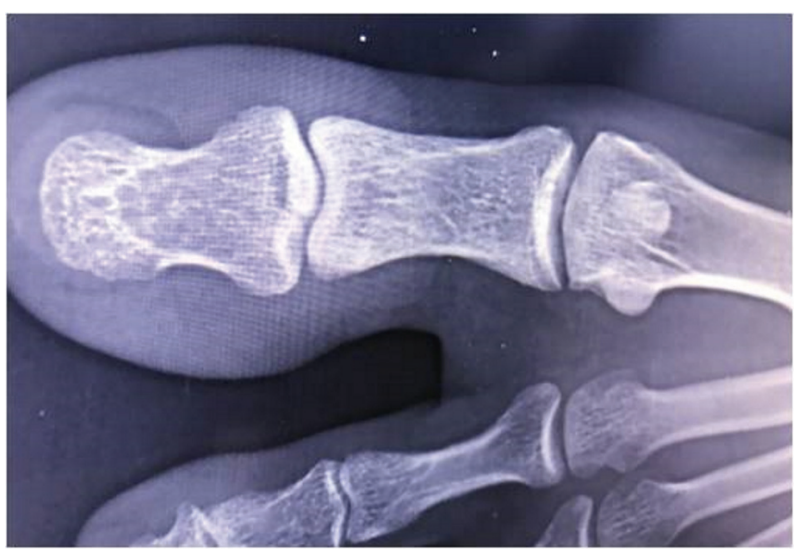

Figure 2. X-ray AP view showing fracture of distal phalanx.

could not practice karate. On the second day he had persistent pain and swelling and bruise around the IP joint of the left great toe. Then he consulted with an orthopedic surgeon with an X-ray film of the affected site which revealed avulsion fracture of distal phalanx due to pulling of extensor hallucis longus (EHL) tendon but no mallet deformity (Figs. 1, 2). As the fracture involved the distal phalanx, it was difficult to stabilize with plaster. So, a mallet splint was applied (Fig. 3) and he was advised for movement restriction for 3 weeks. With the splint and rest, persistent pain was controlled. Repeat X-ray of that foot after 4 weeks revealed a little gap between the fragments but possibly the bone piece was hooked with the joint capsule and soft tissue and no mallet deformity was noted (Fig. 4). The surgeon could resume regular operation after 2 weeks and practice karate after the fourth week.

\section{Discussion}

Toe phalangeal fractures are commonly encountered in orthopedic outpatient department (OPD) comprising 3.6-8\% of all lower extremity injuries [3, 4]. Most fracture of the hallux occurs at the distal phalanx and is most commonly a result of a

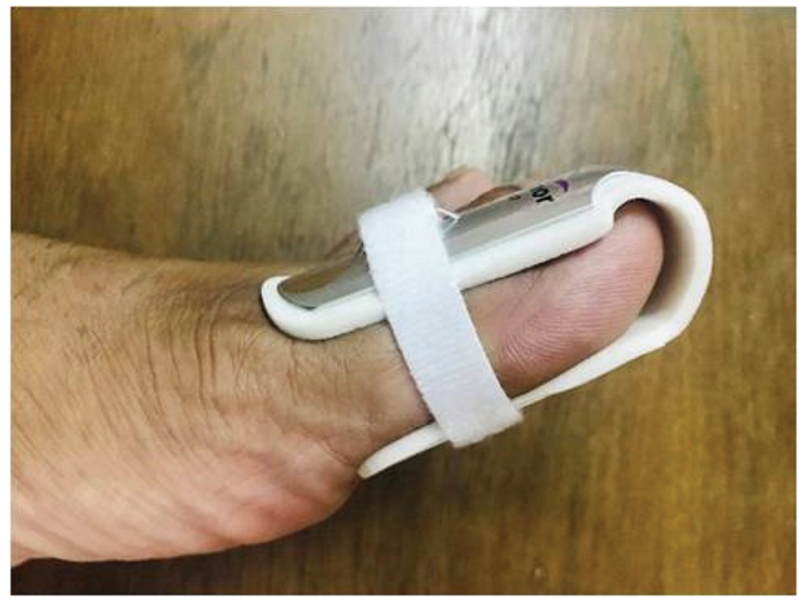

Figure 3. Left hallux immobilized with mallet splint.

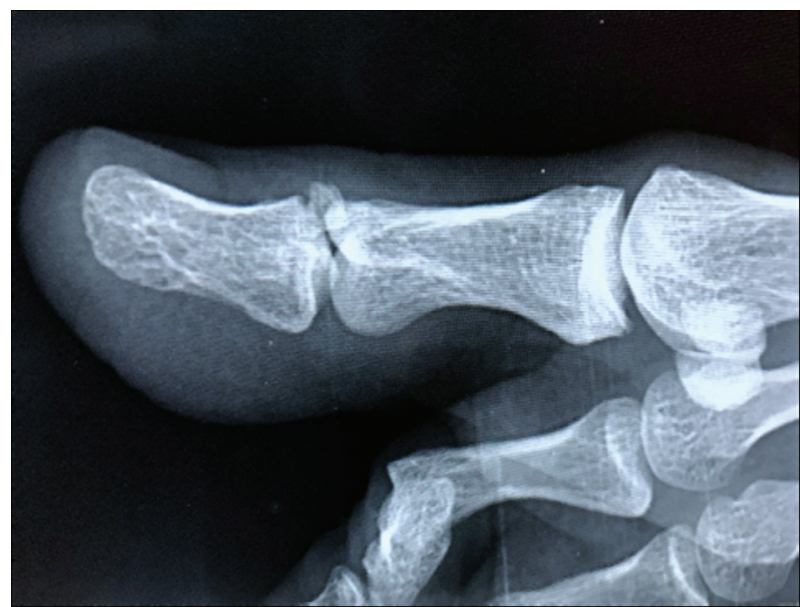

Figure 4. X-ray after 4 weeks showing a little gap between the bone fragments.

direct crushing type injury. Unfortunately, the greatest proportion of all toe phalangeal fracture comprises the hallux (38$56 \%$ ) $[5,6]$. But there are no evidence-based guidelines regarding the indication of surgery in closed phalangeal fracture [6].

A recent review of 707 patients of toe phalangeal fractures seen in fracture clinic in London over a 1-year period reported that only two of them required surgery for loss of articular congruency or deformity. It is suggested that the ones who displaced more than $2 \mathrm{~mm}$, involving intra-articular component, physeal fracture or open fracture, require referral to an orthopedic surgeon [6]. Successful treatment can be achieved with immobilization with external splinting for 6 - 10 weeks and most commonly reported complications were flexion deformity, and restricted motion of IP joint $[7,8]$.

Forceful violent hyperflexion of the hallux IP joint can cause disruption of EHL tendon with mallet deformity of avulsion of a piece of distal phalanx. Here our patient developed an avulsion fracture and remained unnoticed and unsupported for 2 days which was vulnerable for further injury, inflammation and future complications.

\section{Conclusions}

It is not always possible to take precaution from susceptible sports injuries. However, any bone or joint pain while playing sports should not be overlooked. Timely radiological assessment with adequate rest and proper immobilization is the key to effective treatment and avoiding complications.

\section{References}

1. Morrison W. Avulsion fracture: What you need to know. 2017. Retrieved from https://www.medicalnewstoday. com/articles/319471.

2. Schiller J, DeFroda S, Blood T. Lower extremity avulsion fractures in the pediatric and adolescent athlete. J Am 
Acad Orthop Surg. 2017;25(4):251-259.

3. Shibuya N, Davis ML, Jupiter DC. Epidemiology of foot and ankle fractures in the United States: an analysis of the National Trauma Data Bank (2007 to 2011). J Foot Ankle Surg. 2014;53(5):606-608.

4. Court-Brown CM, Caesar B. Epidemiology of adult fractures: A review. Injury. 2006;37(8):691-697.

5. Van Vliet-Koppert ST, Cakir H, Van Lieshout EM, De Vries MR, Van Der Elst M, Schepers T. Demographics and functional outcome of toe fractures. J Foot Ankle Surg. 2011;50(3):307-310.

6. Eves TB, Oddy MJ. Do broken toes need follow-up in the fracture clinic? J Foot Ankle Surg. 2016;55(3):488-491.

7. Hennessy MS, Saxby TS. Traumatic 'mallet toe' of the hallux: a case report. Foot Ankle Int. 2001;22(12):977978.

8. Rapoff AJ, Heiner JP. Avulsion fracture of the great toe: a case report. Foot Ankle Int. 1999;20(5):337-339. 